\title{
FACTORES GENÉTICOS ASOCIADOS A LA HIPOMINERALIZACIÓN INCISIVO-MOLAR. REVISIÓN DE LITERATURA
}

\author{
GENETIC FACTORS ASSOCIATED WITH INCISOR-MOLAR HYPOMINERALIZATION. \\ LITERATURE REVIEW
}

María Cristina Hinostroza Izaguirre

mar_crist22@hotmail.com

ORCID: 0000-0002-5485-6846

Rossmary Janelle Navarro Beteta ${ }^{1}$

rossmary.navarro@gmail.com

ORCID: 0000-0003-2468-0138
Dessire Milagros Abal Perleche ${ }^{1}$

dessiremily@gmail.com

ORCID: 0000-0002-5420-4709

Guido Perona Miguel de Priego ${ }^{1}$

guidoperona54@gmail.com

ORCID: 0000-0003-4092-7364

\section{RESUMEN}

La hipomineralización del incisivo molar (HIM) es un defecto cualitativo del esmalte dental que afecta a los primeros molares permanentes $\mathrm{y}$, a menudo, en asociación con los incisivos permanentes. Con respecto a su etiología, algunos factores pueden estar asociados a su desarrollo, tales como complicaciones prenatales, perinatales y posnatales, y actualmente se considera la predisposición genética. El control genético del desarrollo dental representa una serie compleja de eventos y las mutaciones ocasionales en los genes que codifican las proteínas del esmalte pueden causar alteraciones que afectan las vías moleculares, es por ello que la amelogénesis está bajo un control genético estricto. Actualmente, existe poca evidencia sobre la influencia de las variaciones genéticas en los genes que codifican las proteínas de la matriz del esmalte en la aparición de defectos del esmalte del desarrollo. Por lo tanto, el propósito de esta revisión de la literatura es dar a conocer una revisión actualizada sobre los factores genéticos asociados a la aparición de hipomineralización incisivo-molar.

Palabras clave: genética, esmalte dental, dentición permanente, diente primario

\section{ABSTRACT}

Molar incisor hypomineralization $(\mathrm{MIH})$ is a qualitative defect of the tooth enamel that affects the first permanent molars, often in association with the permanent incisors. Some factors may be associated with the etiology of $\mathrm{MIH}$, including prenatal, peri-natal and postnatal complications and genetic predisposition. Genetic control of dental development involves a complex series of events, and occasional mutations in the genes that encode enamel proteins can cause alterations that affect molecular pathways, which is why amelogenesis is under strict genetic control. Currently, there is little evidence of the influence of genetic variations in the

Citar como: Hinostroza-Izaguirre MC, Abal-Perleche DM, Navarro-Beteta RJ, Perona-Miguel de Priego G. Factores genéticos asociados a la hipomineralización incisivo-molar. Revisión de literatura. Rev Cient Odontol (Lima). 2019; 7 (1): 148-156. 
genes encoding enamel matrix proteins on the appearance of developmental enamel defects. Therefore, the purpose of this literature review is to provide an updated review on the genetic factors associated with the appearance of $\mathrm{MIH}$.

Keywords: genetics, dental enamel, dentition permanent, tooth deciduous

\section{INTRODUCCIÓN}

La etiología de la hipomineralización incisivo-molar (HIM) ha sido muy estudiada; sin embargo, según la evidencia científica, no existen factores asociados (prenatales, perinatales, posnatales, genéticos ni ambientales) específicos a su aparición.

La hipomineralización incisivo molar es el término utilizado para describir una alteración específica causada por un disturbio durante la fase inicial de la maduración del esmalte en los primeros molares permanentes, que puede estar asociados a los incisivos ( ${ }^{1}$ ). Clínicamente, presenta una severidad asimétrica con opacidades demarcadas que varían en el tono de color de blanco a amarillo/marrón, con una demarcación marcada entre el esmalte afectado y el esmalte sano $\left({ }^{2}\right)$.

La HIM se describió originalmente como un defecto idiopático y aún no se ha definido una etiología clara de la enfermedad $\left({ }^{3,4}\right)$. Esta condición se ha asociado con una variedad de factores etiológicos, pero, según los resultados de dos revisiones sistemáticas, ninguno de los posibles factores de riesgo analizados presentó una causalidad convincente $\left({ }^{4,5}\right)$.

Actualmente, existen estudios que relatan la relación entre los genes y el HIM, los cuales cumplen una función durante la formación del esmalte; es por ello que se podría decir que hay una estrecha relación entre la genética y la aparición de HIM. Los datos disponibles muestran principalmente asociaciones entre diferentes variaciones genéticas en genes que codifican proteínas del esmalte dental y diversas alteraciones en el desarrollo del diente.

Además, es posible que las variaciones genéticas en los genes que codifican las proteínas del esmalte también puedan interactuar de alguna manera con los factores ambientales $\left({ }^{6,7}\right)$. De acuerdo con lo relatado, el propósito de esta revisión de la literatura es dar a conocer una revisión actualizada sobre los factores genéticos asociados a la aparición de hipomineralización incisivo-molar.

Se realizó una búsqueda bibliográfica para identificar los artículos utilizados en esta revisión de literatura. Las bases de datos incluyeron diferentes idiomas. Las publicaciones en inglés y español fueron obtenidas de Pubmed, Medline, Scielo, y se utilizaron diferentes combinaciones de las palabras claves (hypomineralization, genetics), que arrojaron 19 artículos fechados entre los años 1996 y 2019.

Las listas de referencias identificadas se verificaron por duplicación, en cuyo caso fueron eliminadas; se seleccionaron los títulos y los resúmenes de los artículos identificados en la lista potencialmente elegible. Las publicaciones en las que no se estudiaron la asociación de los factores genéticos con la hipomineralización incisivo-molar se excluyeron a través de la selección de títulos y resúmenes.

\section{AMELOGÉNESIS}

El proceso de formación de las piezas dentarias se origina por la interacción entre el epitelio oral y la ectomesenquima, a través de una serie de eventos temporales y altamente regulados por un 
proceso denominado amelogénesis $\left(^{(8)}\right.$. La amelogénesis comienza con la formación del esmalte donde los ameloblastos depositan una matriz orgánica enriquecida con proteínas durante la etapa de secreción. Esta matriz proteica se modifica luego a través de las etapas de transición y maduración $\left({ }^{9}\right)$. Durante la etapa secretora, los ameloblastos en forma de columnas polarizadas adyacentes a la formación del esmalte secretan proteínas como la amelogenina y la ameloblastina. Los procesos de Tomes en la superficie distal de los ameloblastos secretores organizan la dirección de la deposición del esmalte en barras $\left({ }^{10}\right)$. Durante las etapas de maduración y mineralización, las proteínas de la matriz del esmalte se degradan de manera gradual mediante la metaloproteinasa-20 de la matriz (MMP20) y la calicreína-4 (KLK-4) para formar prismas de esmalte únicos ${ }^{(11,12)}$.

Se pueden producir diferentes tipos de defectos del esmalte según la etapa de desarrollo afectada. El defecto dental conocido como hipoplasia del esmalte es el resultado de la reducción del grosor del esmalte, que se produce durante la fase secretora de la amelogénesis. Cuando los ameloblastos se ven afectados en la etapa tardía de la mineralización o maduración de la amelogénesis, puede ocurrir un defecto en la translucidez del esmalte. Estos defectos se denominan hipomineralización del esmalte $\left.{ }^{(13,14}\right)$.

Teniendo en cuenta que los ameloblastos son células muy sensibles y que la amelogénesis está controlada genéticamente, la susceptibilidad genética puede estar asociada con la patogénesis de la MIH. Se ha observado una asociación entre las variaciones en los genes AMBN, ENAM, TUFT1, TFIP11 y SCUBE1, y una mayor susceptibilidad a MIH; sin embargo, los factores ambientales que actúan durante la formación del esmalte también pueden interferir con la función de las proteínas expresadas por estos genes $\left(^{2}\right)$.
El control genético del desarrollo dental representa una serie compleja de eventos, y las mutaciones ocasionales en los genes que codifican las proteínas del esmalte pueden causar alteraciones que afectan las vías moleculares. Por lo tanto, la amelogénesis está bajo un control genético estricto e incluso la susceptibilidad a la caries puede verse afectada por la variación genética $\left(^{8}\right)$.

\section{ETIOLOGÍA}

Aún no existen datos concluyentes sobre la etiología de esta hipomineralización. Sin embargo, algunos factores pueden estar asociados al desarrollo de la HIM, como complicaciones pre, peri y posnatales: infecciones urinarias, parto pretérmino, hipoxia, enfermedades gastrointestinales y del sistema respiratorio, fiebres altas y, actualmente, también se ha considerado la predisposición genética $\left({ }^{4,15,16}\right)$.

\section{PREVALENCIA}

La prevalencia parece variar según el país, la región o el grupo de edad considerado, y aún es difícil juzgar si la HIM está aumentando. La prevalencia informada de HIM varía del 2,4\% en Bulgaria y Alemania, el $13,9 \%$ en Noruega y el $17 \%$ en Finlandia al $37,3 \%$ en Dinamarca $y$ el $40,2 \%$ en Brasil (18-23).

\section{GENÉTICA}

Es importante mencionar los conceptos siguientes:

- Fenotipo. Conjunto de caracteres visibles que un individuo presenta como resultado de la interacción entre sus genes y el medio ambiente. 
- Cromosoma. Estructura que se encuentra en el núcleo de las células y que transportan fragmentos largos de ADN. Los cromosomas vienen en pares. Normalmente, cada célula en el cuerpo humano tiene 23 pares de cromosomas. Los cromosomas restantes se denominan autosómicos y se conocen como pares del 1 al 22.

- Gen. Unidad fundamental, física y funcional de la herencia, que transmite información de una generación a la siguiente. Los genes están organizados en segmentos en el ADN uno detrás de otro; la información genética que está codificada en el ADN tiene que pasar por una estructura de la célula (ribosoma) que convierte la información genética en proteínas.

\section{GENÉTICA DEL DESARROLLO DEL ESMALTE}

Se ha observado una asociación entre las variaciones de los genes que forman el esmalte, como AMTN (amelotin), AMBN (ameloblastina - cromosoma 4), ENA (enamelisin, cromosoma 11) ENAM (enamelina), TUFT1 (tuftelina - cromosoma 1), TFIP11, KLK4 (calicreína 4), LM-332 (laminin y2) y SCUBE1 (24-27).

Jeremías et al. (2016) realizaron un estudio en el cual determinaron que los disturbios genéticos durante el desarrollo dental influyen en la variación del número y tipo de dentición. El estudio menciona 11 marcadores en 5 genes [ameloblastina (AMBN), amelogenina (AMELX), enamelina (ENAM), tuftelina (TUFT1) y proteína de interacción tuftelina 11 (TFIP11)], que tuvieron como resultado que el marcador ENAM fue asociado al HIM. Cuando los ameloblastos son afectados en la fase tardía de la amelogénesis de mineralización o maduración, puede ocurrir un defecto en la traslucidez del esmalte. AMELX y ENAM son dos genes cuyos marcadores pueden conducir amelogénesis imperfecta.

El $97 \%$ de las personas afectadas por HIM portan el alelo rs5979395*G. Debido a que, aproximadamente, entre el $5 \%$ y el $10 \%$ de todos los casos de amelogénesis imperfecta están ligados al cromosoma $X$, es predecible encontrar una mutación en el gen AMELX. Algunos estudios demuestran que la variación genética en este gen está asociada no solo con la amelogénesis imperfecta, sino también con la HIM. Este gen es fundamental para la amelogénesis, que codifica la amelogenina, la proteína principal del esmalte dental secretado por los ameloblastos durante la etapa de secreción de la amelogénesis $\left({ }^{28}\right)$.

También se ha sugerido que la tuftelina (TUFT1, 1q21) juega un papel importante durante el desarrollo del esmalte y la mineralización $\left({ }^{29}\right)$.

Según estudios, la HIM es un trastorno multifactorial porque diferentes genes pueden influir en su aparición. Además, existe evidencia sustancial que apoya la participación de los componentes epigenéticos en la definición de las variaciones fenotípicas humanas. La epigenética describe la forma en que las interacciones gen-ambiente y gen-gen dan forma a un fenotipo durante el desarrollo. Actualmente, la epigenética describe alteraciones en la función genómica, principalmente cambios hereditarios mitóticamente en la expresión genética, que ocurren a través de modificaciones químicas reversibles en la estructura de la cromatina sin alterar la secuencia del ADN $\left({ }^{28}\right)$.

\section{FENOTIPO DE LA HIPOMINERALIZACIÓN}

La HIM es un defecto cualitativo del esmalte que se da por una pobre 
mineralización del esmalte en desarrollo. Se puede visualizar como una opacidad blanco cremoso, marrón amarillento con o sin degradación poseruptiva. La HIM se puede dar en primeros molares permanentes y, con frecuencia, también puede afectar a los incisivos permanentes $\left({ }^{30}\right)$.

Según el criterio EAPD (2003), solo se pueden informar dos fenotipos: $\mathrm{MH}$ y $\mathrm{M}+\mathrm{IH}$. Otro fenotipo informado en la dentición permanente es la hipomineralización del incisivo idiopático, la cual afecta solo a los incisivos permanentes. Sin embargo, la HIM puede afectar a otros dientes como los primarios y caninos permanentes, premolares y segundos molares.

Hay pocos estudios sobre hipomineralización en segundos molares deciduos y se ha propuesto el término hipomineralización molar decidua $(\mathrm{DMH})$ para describir este fenotipo.

Existe un esquema de clasificación para la hipomineralización del esmalte (fig. 1):
1. Tipo I (MH): hipomineralización del esmalte que afecta solo a los primeros molares permanentes.

2. Tipo II (HI): hipomineralización del esmalte que afecta solo a los incisivos permanentes.

3. Tipo III $(M+l H)$ : hipomineralización del esmalte que afecta a los primeros molares e incisivos permanentes.

4. Tipo IV (MIHO): hipomineralización del esmalte que afecta al menos a uno de los caninos o premolares, o al segundo molar con una participación concomitante de al menos un primer molar permanente; y los incisivos permanentes pueden verse afectados simultáneamente.

5. Tipo V (NoFPM): hipomineralización del esmalte que afecta al menos a uno de los caninos o premolares, o al segundo molar, pero no a los primeros molares permanentes, y los incisivos permanentes pueden verse afectados simultáneamente $\left.{ }^{31}\right)$.

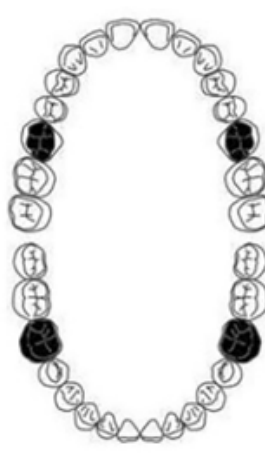

Type l: MH

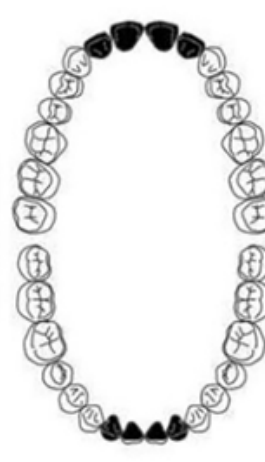

Type II: IH

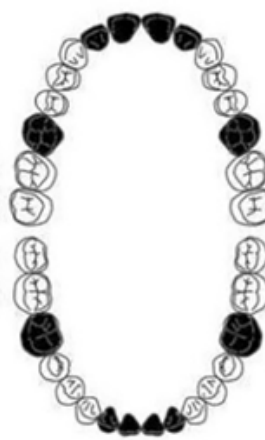

Type III: MIH

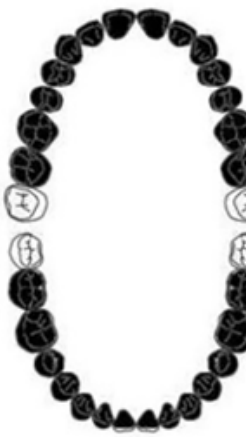

Type IV: Miно

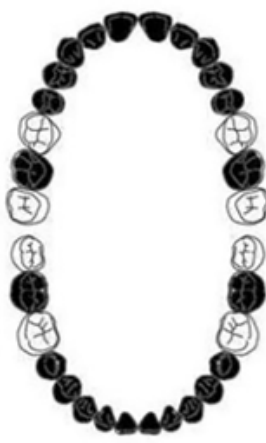

Type V: NoFPM

Figura 1. Fenotipos de la hipomineralización (tomado de Lygidakis NA,Wong F, JäLeVIK B, ET AL. ${ }^{31}$ )

El estudio de Mitall (2016) encontró que la prevalencia de hipomineralización del esmalte fue del $13,21 \%$ y la prevalencia de HIM (hipomineralización por FPM, es decir, fenotipos $\mathrm{MH}, \mathrm{M}+\mathrm{IH}$ y $\mathrm{MIHO}$ ) fue del $9,79 \%$. Se identificaron cinco fenotipos diferentes que variaron en extensión y gravedad de la hipomineralización del esmalte. El fenotipo MIHO presenta mayor gravedad y el fenotipo IH fue el de menor gravedad. Este es el primer estudio que informa sobre la extensión y la severidad de la hipomineralización del esmalte en el complemento completo de la dentición 
permanente utilizando los criterios de EAPD 2003. Se deben realizar estudios futuros para informar sobre la extensión y las manifestaciones de diversos fenotipos en dentición permanente, dentición mixta y dentición primaria $\left({ }^{32}\right)$.

\section{CONCLUSIONES}

1. Existe diversos genes que están implicados durante la amelogénesis cumpliendo cada uno su función correspondiente, por lo que existe un fuerte control genético dentro de ella.

2. Según la evidencia científica, se ha podido mostrar la influencia de la genética en la aparición de HIM. Sin embargo, estos estudios sugieren la realización de más investigaciones que incluyan poblaciones étnicas más grandes, bien diagnosticadas y diferentes para expandir la investigación de los factores genéticos y ambientales, así como el entorno genético e interacciones que puedan influir en la aparición del HIM.

Contribución del autor: María Cristina Hinostroza Izaguirre, Dessire Milagros Abal Perleche, Rossmary Janelle Navarro Beteta han participado en la concepción del artículo, la recolección de información, su redacción y aprobación de la versión final. Guido Perona Miguel del Priego participó en la concepción del artículo y aprobación de la versión final.

Fuente de financiamiento: Autofinanciado.

Conflicto de interés: Los autores declaran no tener conflicto de interés de ningún tipo. 


\section{REFERENCIAS BIBLIOGRÁFICAS}

1. Weerheijm KL, Duggal M, Mejàre I, Papagiannoulis L, Koch G, Martens LC, et al. (2003). Judgement criteria for molar incisor hypomineralisation (MIH) in epidemiologic studies: a summary of the European meeting on MIH held in Athens, 2003. Eur J Paediatr Dent 4: 110-3.

2. Barbosa $\mathrm{R}$ et al. Exploring the association between genetic and environmental factors and molar incisor hypomineralization: evidence from a twin study. International Journal of Paediatric Dentistry. 2017; 28 (2): 198-206.

3. Weerheijm KL. Molar incisor hypomineralization (MIH). Eur J Pediatr Dent 2003; 4: 114-120.

4. Alaluusua S. Aetiology of molar-incisor hypomineralization: a systematic review. Eur Arch Paediatr Dent 2010; 11: 53-8.

5. Crombie F, Manton D, Kilpatrick N. Aetiology of molar-incisor hypomineralization: a critical review. Int J Pediatr Dent 2009; 19: 73-83.

6. Gerreth K, Zaorska K, Zabel M, Nowicki M, Borysewicz-Lewicka M. Significance of genetic variations in developmental enamel defects of primary dentition in Polish children. Clin Oral Invest.2018; 22: 321-9.

7. Kühnisch J, Heitmüller D, Thiering $\mathrm{E}$, Brockow I, Hoffman U, Neumann C, et al. Proportion and extent of manifestation of molar-incisor hypomineralizations according to different phenotypes. Journal of Public Health Dentistry. 2014; 74(1):42-9.

8. Jeremias F, et al. Family-based genetic association for molar-incisor hypomineralization. Caries Res. 2016; 50: 310-8.

9. Simmer JP, Richhardson AS, Hu YY, Smith CE, Ching-Chung Hu J. A post-classical theory of enamel biomineralization and why we need one. Int. J Oral Sci. 2012; 4: 129-34.

10. Simmer JP, Papagerakis P, Smith CE, Ficher DC, Rountrey AN, Zheng L, Hu JC. Regulation of dental enamel shape and hardness. J Dent Res. 2010; 89: 1024-38.

11. Bartlett JD, Simmer JP. Kallikrein-related peptidase-4 (KLK4): role in enamel formation and revelations from ablated mice. Front Physiol. 2014; 5: 240.

12. Bartlett JD, Skobe Z, Nanci A, Smith CE. Matrix metalloproteinase 20 promotes a smooth enamel surface, a strong dentino-enamel junction, and a decussating enamel rod pattern. Eur J Oral Sci. 2011; 119: 199-205.

13. Suckiling GW. Developmental defects of enamel-historical and present-day perspectives of their pathogenesis. Adv Dent Res. 1989; 3 (2): 87-94.

14. Seow WK. Clinical diagnosis of enamel defects: Pitfalls and practical guidelines. Int Dent J. 1997; 47 (3): 173-82.

15. Elhennawy K, Schwendicke F. Managing molar-incisor hypomineralization: A systematic review. J Dent. 2016; 55: 16-24. 
16. Tourino LF, Corrêa-Faria P, Ferreira RC, Bendo CB, Zarzar PM. Association between molar incisor hypomineralization in schoolchildren and both prenatal and postnatal factors: a population-based study. PLoS One. 2016; 11 (6): e0156332.

17. Denis M, Atlan A, Vennat E, Tirlet G, Attal JP. White defects on enamel: diagnosis and anatomopathology: two essential factors for proper treatment. Part 1. Int Orthod 2013; 11: 139-65.

18. Kukleva MP, Petrova SG, Kondeva VK, Nihtyanova TI. Molar incisor hypomineralization in 7- to 14-year-old children in Plovdiv, Bulgaria- an epidemiologic study. Folia Med (Plovdiv) 2008; 50: 71-5.

19. Dietrich G, Sperling S, Hetzer G. Molar incisor hypomineralization in a group of children and adolescents living in Dresden (Germany). Eur J Paediatr Dent 2003; 4: 133-7.

20. Schmalfuss A, Stenhagen KR, Tveit AB, Crossner CG, Espelid I. Canines are affected in 16-yearolds with molar-incisor hypomineralization (MIH): an epidemiological study based on the Troms $\varnothing$ study: 'Fit Futures'. Eur Arch Paediatr Dent. 2015. Epub ahead of print.

21. Alaluusua S, Lukinmaa PL, Koskimies M, Pirinen S, Hölttä P, Kallio M, Holttinen T, Salmenperä L. Developmental dental defects associated with long breast feeding. Eur J Oral Sci 1996; 104: 493-7.

22. Wogelius P, Haubek D, Poulsen S. Prevalence and distribution of demarcated opacities in permanent 1st molars and incisors in 6- to 8-year-old Danish children. Acta Odontol Scand 2008; 66: 58-64.

23. Soviero V, Haubek D, Trindade C, Matta T, Poulsen S. Prevalence and distribution of demarcated opacities and their sequelae in permanent 1st molars and incisors in 7 to 13-year-old Brazilian children. Acta Odontol Scand 2009; 67: 170-5.

24. Nunez S, Chun Y, Ganss B, Hu Y, Richardson A, Schmitz J, et.al. Maturation stage enamel malformations in Amtn and Klk4 null mice. Matrix Biol. 2016; 52-54: 219-33.

25. Wazen R, Viegas-Costa L, Fouillen A, Moffatt P, Adair-Kirk T, Senior R, et.al. Laminin y2 Knockout mice rescued with the human protein exhibit enamel maturation defects. Matriz Biol. 2016; 52-54: 207-18.

26. Teixeira R, Andrade N, Queiroz L, Mendes F, Moura M, Moura L, et.al. Exploring the association between genetic and enviorenmental factors and molar incisor hypomineralization: evidence from a twin study. Int J Paediatr Dent. 2018; 28 (2): 198-206.

27. Kuhnisch J, Thiering E, Heitmuller D, Tiesler C, Grallert $\mathrm{H}$, Heinrich-Weltzien R, Hickel R, et.al. Genome-wide association study (GWAS) for molar-incisor hypomineralization (MIH). Clin Oral Investig. 2014; 18 (2): 677-82.

28. Jeremias F, et al. Family-based genetic association for molar-incisor hypomineralization. Caries Res 2016; 50: 310-8.

29. Deutsch $D$, et al. The human tuftelin gene and the expression of tuftelin in mineralizing and nonmineralizing tissues. Connect Tissue Res 2002; 43: 425-34. 
María Cristina Hinostroza Izaguirre, Dessire Milagros Abal Perleche,

Rossmary Janelle Navarro Beteta

30. Weerheijm KL, Jalevik B, Alaluusua S. Molar-Incisor Hypomineralisation. Caries Res. 2001; 35: 390-1.

31. Lygidakis NA,Wong F, Jälevik B, et al. Best clinical practice guidance for clinicians dealing with children presenting with molar-incisor-hypomineralization $(\mathrm{MIH})$. Eur Arch Paediatr Dent. 2010; 11: 75-81.

32. Mittal N. Phenotypes of enamel hypomineralization and molar incisor hypomineralization in permanent dentition: identification, quantification and proposal for classification. J Clin Pediatr Dent. 2016; 40 (5): 367-74.

ARTículo RECIBIDO: 18-04-2019

ARTículo ACEPTADO: 27-05-2019 